


Aqueous

traction

REE:

LREES

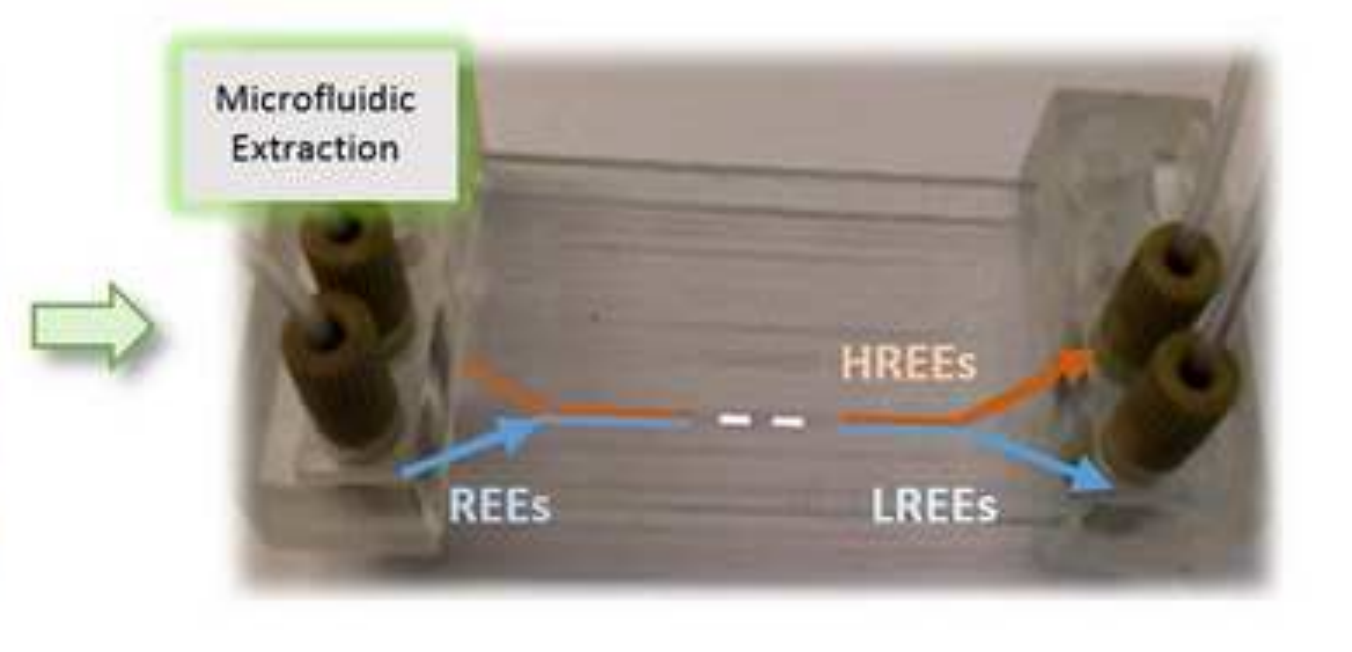




\title{
Microfluidic Solvent Extraction of Rare Earth Elements from a Mixed Oxide Concentrate Leach Solution using Cyanex ${ }^{\circledR} 572$
}

\author{
Elisabeth Kolar ${ }^{1, *}$, Rik P. R. Catthoor ${ }^{1, *}$, Frederik H. Kriel ${ }^{1}$, Rossen Sedev ${ }^{1}$, Scott Middlemas ${ }^{2}$, Eric Klier ${ }^{2}$, \\ Gareth Hatch ${ }^{3}$, and Craig Priest ${ }^{1 *}$ \\ ${ }^{1}$ Future Industries Institute, University of South Australia, Mawson Lakes, SA 5095, Australia \\ ${ }^{2}$ Army Research Laboratory, Aberdeen Proving Ground, Aberdeen, MD 21005, United States \\ ${ }^{3}$ Technology Metals Research, LLC, 180 S. Western Ave 150, Carpentersville, IL 60110, United States \\ *Corresponding author: craig.priest@unisa.edu.au \\ ${ }^{*}$ These authors contributed equally to this publication
}

\begin{abstract}
Solvent extraction of rare earth elements (REEs) involves hundreds of individual extraction and phase separation cycles, fine adjustment of solution conditions, and individual stage and overall process times that are long. Therefore, we investigated microfluidic solvent extraction (microSX) of REEs from a leached mixed rare earth oxide (REO) mineral concentrate using a phosphorus-based cationic exchange extractant (Cyanex ${ }^{\circledR}$ 572). A Y-Y microchip was used, in which the aqueous and organic phases were contacted for up to $15 \mathrm{~s}$ with sub-second resolution. The extraction rate and selectivity for heavy REEs was determined for the prepared leach solution. Good selectivity for heavy REEs was observed using the microchip for leach solutions adjusted to $\mathrm{pH}$ 0.7. Extraction rates on the microchip were typically double that observed in conventional (bulk) solvent extractions, except for $\mathrm{Lu}$ and $\mathrm{Yb}$, which were three-times faster. The faster extraction can be largely attributed to the higher surface-to-volume ratio achieved in our microfluidic experiments; double that observed for bulk extractions under the conditions employed.
\end{abstract}

\section{Keywords:}

Rare Earth Elements, Solvent Extraction, Microfluidics, Leachate, Extraction Rate, Cyanex ${ }^{\circledR} 572$. 


\section{Introduction}

Rare earth elements (REEs) have been called the metals of technology due to their numerous applications in advanced material science, including phosphors, lasers, ceramics, electronics, magnets, catalysis and many more [1, 2]. REEs are in fact fairly abundant in the Earth's crust but are rather dispersed and their difficult separation is a major challenge. Liquid-liquid solvent extraction is a key step in purifying REEs and is the focus of this paper. Due to the rapidly increasing demand for REEs in technology industries, REE separation and security of the supply chain have become topics of international importance [3]. Novel technologies that can achieve fast, efficient, and selective separation and purification of REEs have the potential to play a lead role in delivering REEs to global technology industries on-time and with high purity.

Minerals containing REEs include bastnaesite $(\mathrm{La}, \mathrm{Ce}) \mathrm{FCO}_{3}$, monazite $(\mathrm{Ce}, \mathrm{La}, \mathrm{Y}, \mathrm{Th}) \mathrm{PO}_{4}$ and xenotime $\mathrm{YPO}_{4}$, and are concentrated by flotation, magnetic or gravity methods followed by leaching with inorganic acids, e.g. $\mathrm{HCl}$ [2]. After leaching, solvent extraction using various extractants (anion/cation exchangers or chelating extractants) is often used to separate and purify the individual REEs. This separation process includes intermediate steps in which groups of REEs are separated, for example heavy and light REEs may be 'split' [2]. Generally, the heavy REEs (HREEs), i.e. from Gd to Lu, and $Y$ are preferentially extracted leaving the lights, i.e. La to $\mathrm{Sm}$, behind $[4,5]$. For the extraction of HREEs, organophosphorus acidic cation extractants are used, such as di(2-ethylhexyl)phosphoric acid (P204), 2-ethylhexylphosphonicmono-2-ethylhexyl ester (P507) and bis(2,4,4-trimethylpentyl) phosphinic acid (Cyanex ${ }^{\circledR}$ 272). A recent addition to this class of extractants is Cyanex ${ }^{\circledR} 572$ (C572, Cytec)[6], which is specially formulated for extraction of the HREEs with lower acid requirement during back extraction and stripping [7].

The extraction of REEs from aqueous to organic phase proceeds via cation exchange with an extractant as follows:

$$
\mathrm{Ln}^{3+}+3 \overline{\mathrm{HA}} \rightleftharpoons \overline{\mathrm{LnA}_{3}}+3 \mathrm{H}^{+}
$$


where Ln represents the REE, HA is the extractant, and the overscoring denotes the organic phase [2]. Thus, the $\mathrm{pH}$ of the aqueous phase can be used to manipulate the equilibrium of the ion exchange. The $\mathrm{pH}$ at which a given extraction (e.g. $50 \%$ ) occurs decreases as the atomic number of the REE increases thus allowing a degree of selectivity. Furthermore, more acidic aqueous phases can be used to strip REEs from a loaded organic phase downstream of the extraction.

One of the most common bulk solvent extraction (bulk SX) methods employs a mixer-settler, the aqueous and organic phases are combined and vigorously stirred to form an emulsion.[8] The high surface-to-volume ratio of the emulsion increases the rate of extraction/stripping because of the larger interfacial area available for ion exchange and the shorter diffusion lengths involved. At equilibrium, mixing is stopped and the two phases are separated by coalescence and the difference in the density of the two phases. Bulk SX is highly effective for many extraction systems including extraction from mineral leach solutions. The extraction of REEs however, requires hundreds of extractions [4], scrubbing, and stripping stages, resulting in REE refineries with large physical footprints and long processing times. Furthermore, the presence of fine particles (e.g. silica) and surfactants in a mineral leach solution can slow down the extraction by limiting the availability of the interface for extraction and/or by forming an emulsion stabilized by fine particles (known as crud) [9]. Crud formation is a major issue as it retards phase disengagement by protecting droplets against coalescence, often for very long periods, leading to unrecoverable losses of REEs or uneconomic throughput [9].

Microfluidic devices are being explored as an alternative approach for solvent extraction, including droplet and stream-based extraction chips [10]. Microfluidic solvent extraction has been reported for liquid-liquid extraction of $\mathrm{Ag}[11]$, $\mathrm{Co}[12,13], \mathrm{Cu}[14-17], \mathrm{Cr}[16], \mathrm{Pt}[18,19], \mathrm{Pd}[19]$, and various lanthanides [20-23] and actinides [24-26]. As with bulk SX, increasing the surface-tovolume (S/V) ratio in microfluidic extraction increases the rate of extraction $[10,19,23,24,27$. However, for microfluidic extraction, it is possible to precisely tune the $S / V$ ratio [10]. The rate of extraction is an important consideration since microSX throughput is linearly related to the contact time required for extraction, assuming that channel dimensions are fixed. With the small cross- 
sectional dimensions of the micro-channels (typically tens of $\mu \mathrm{m}$ ), the $S / V$ ratios are very high and extraction rates are relatively fast $(<10$ s) in many cases $[11,25,26,28]$. This makes it possible to achieve efficient extractions in microchannels that are a few centimetres long at modest flow rates $(0.1-10 \mathrm{~mL} / \mathrm{h})[11,14-16,19]$. However, microSX is not without its challenges. High volumetric throughput will require "numbering-up", i.e. parallel operation of many chips to maintain the optimised (microscale) physical and chemical conditions. This introduces engineering challenges that we have addressed in part elsewhere for the case of platinum extraction [28].

Here, we focus on stream-based extraction chips - specifically the Y-Y chip design [14] - based on our earlier work demonstrating that the absence of droplets avoids crud formation when extracting metals from mineral leach solutions $[14,16]$. We have focused on real mineral leach solutions wherever possible and, in this study, stream-based microfluidic extraction of REE solutions obtained from acid leaching of a REO concentrate is investigated. The primary aim is to demonstrate a suitable 'split' between heavy and light REEs, and investigate rates of extraction with respect to individual elements and the sum of the HREEs present. The results are compared with bulk extractions, revealing higher effective first-order rate constants for extraction in the microchip.

\section{Experimental Section}

\subsection{Preparation of Leach Solution}

To prepare the REO leach solutions, $100 \mathrm{~g}$ of the ore concentrate (Technology Metals Research, USA), see Figure 1(a), was added to $300 \mathrm{~mL}$ of $8 \mathrm{M} \mathrm{HCl}$ and stirred for $24 \mathrm{~h}$. The remaining solids were filtered (Whatman ${ }^{\circledast} \mathrm{Nr} 1$ filter paper) and the clear leach solution, Figure 1(b), was stored at room temperature. The $\mathrm{pH}$ of the filtrate was adjusted to three different values $(3.2,4.0$ and 6.0$)$ using a $30 \%$ ammonium hydroxide solution (APS Chemicals). These solutions were put aside for approximately 3 days to allow iron hydroxide to precipitate. The precipitates were removed by filtration (Whatman ${ }^{\circledR} \mathrm{Nr} 1$ filter paper). The leach solution adjusted to $\mathrm{pH} 3.2$ was the most suitable 
for extraction experiments due to complete removal of iron (see Results and Discussion for details). The $\mathrm{pH}$ was then adjusted to 0.7 to achieve a HREEs/LREEs 'split' before comparative microfluidic, Figure 1(c), and bulk, Figure 1(d), extractions were carried out. ICP-MS (Intertek, Australia) was employed to determine the individual REE concentrations before and after extraction.

\subsection{Extraction}

In all experiments, the extractant was Cyanex ${ }^{\circledR} 572$ (Cytec Industries) and the organic solvent was Shellsol D70 (Shell Chemicals). The concentration of Cyanex ${ }^{\circledast} 572$ was 1 M (approx. 33 v/v \%) to be consistent with that reported in Cytec Industries' product data sheet. Under these conditions, the maximum recommended loading of REE for this organic phase is $30 \%$ of the maximum stoichiometric load, i.e. $0.1 \mathrm{M}$ REE in the organic phase [6]. Bulk solvent extractions were carried out by stirring 25 $\mathrm{mL}$ of organic phase with an impeller at $800 \mathrm{rpm}$ in a $100 \mathrm{~mL}$ beaker while adding an equal volume of loaded aqueous phase. The time required to add the aqueous phase was estimated to be less than 1 $\mathrm{s}$ and the extractions were allowed to run to equilibrium. A plastic Pasteur pipette $(3 \mathrm{~mL})$ was used to remove approximately $1-1.5 \mathrm{~mL}$ samples of the emulsion at various contact times from $5 \mathrm{~s}$ to $5 \mathrm{~min}$. The samples were collected in capped glass vials and briefly allowed to phase separate before removing the aqueous phase to another clean glass vial. The collection time was approx. $2 \mathrm{~s}$ and the phase separation time after collection is estimated to be approx. $10 \mathrm{~s}$. We have ignored these additional periods of liquid-liquid contact when presenting the results of this study. Note that accounting for this in comparisons of extraction rate would favour microSX. Small amounts of entrained organic phase were observed to separate out from the isolated aqueous phase at long time-scales (e.g. 2 - 3 days) forming an organic 'lens' at the surface of the aqueous phase. The volume of this organic phase is no more than $10 \mu \mathrm{L}$, such that any further extraction is likely to be negligible (less than 1\%). 

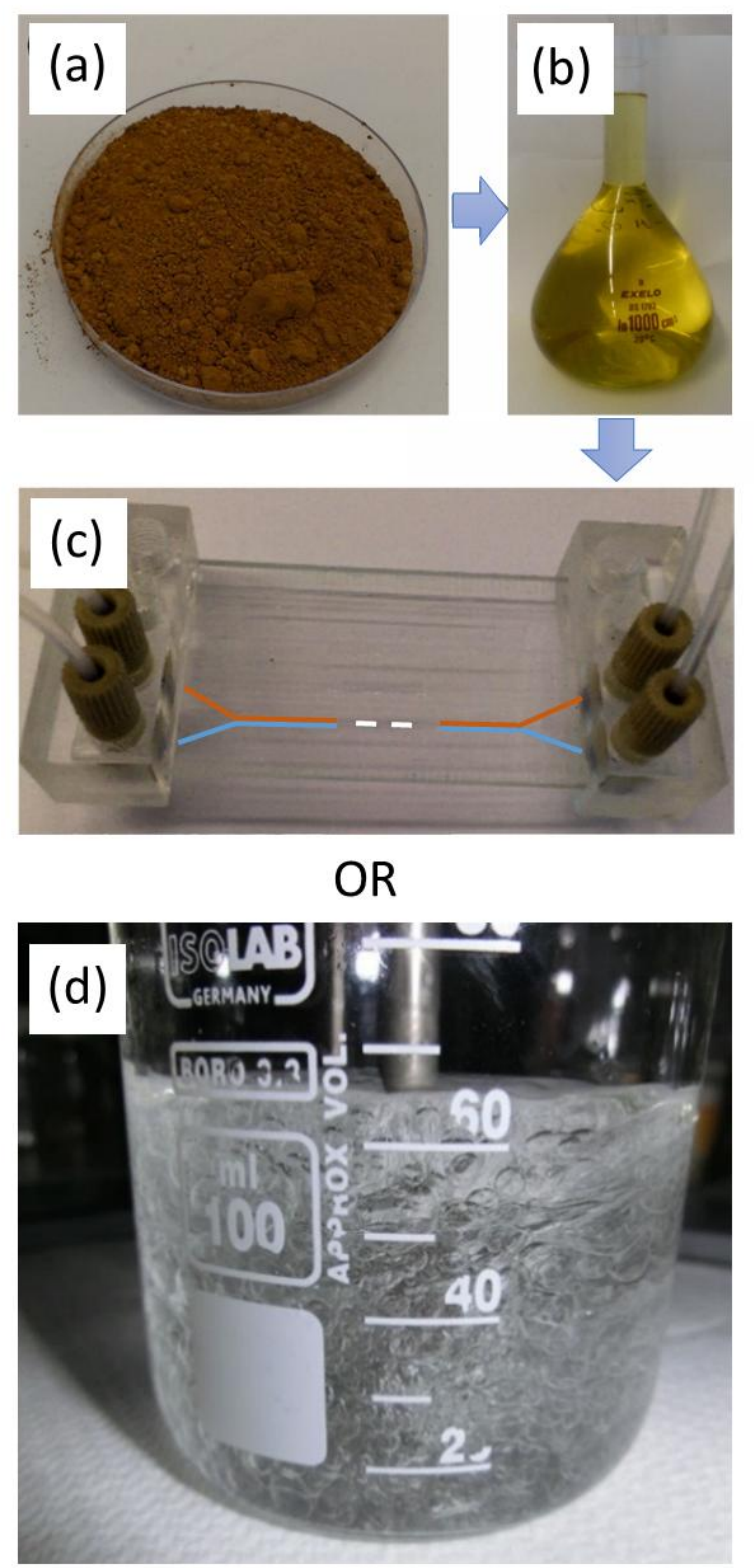

Figure 1. Leaching of a mixed REO concentrate: Images of (a) the REO ore concentrate as supplied, (b) the leach solution ( $\mathrm{pH}$ 3.2), (c) microSX chip in a custom chip-holder with fluid connections, (d) droplets formed during bulk SX in a $100 \mathrm{~mL}$ beaker.

For droplet size analysis in bulk SX, digital images (Casio EX-ZR100, 5 MP, shutter speed priority mode) of the emulsion were collected, as viewed through the wall of the beaker. Due to the small size of the droplets relative to the size of the beaker and imaging only at the centre of the beaker, optical distortion of any individual droplet was minimised. The diameter of more than 100 
droplets was manually measured (as it is common in droplet sizing [29]) for rotating impeller speeds ranging between 400 and $1000 \mathrm{rpm}$.

Micro-solvent extraction experiments were carried out using custom designed borosilicate glass Y-Y microchips (ANFF-SA, Australia) as described previously [16]. The aqueous and organic phases meet at the upstream Y-junction, flow in parallel through the extraction channel, and then separate (the streams diverge) at the downstream Y-junction. Phase separation efficiency has been quantified for platinum extraction and is typically acceptable (1-2\% inefficiency) [18], provided that the microchannel design and flow conditions are well-chosen, see Section 3.2. In this study, phase separation efficiencies were observed to be qualitatively similar. Thus, the two phases are collected separately at the outlet tubing and no significant extraction occurs beyond the downstream $\mathrm{Y}$ junction. Collection times range from tens of minutes to hours, depending on the flow rate used and the amount of sample required for analysis.

To achieve a broader range of contact times, two different length microchips ( $L=129$ or 383 $\mathrm{mm}$ ) were used. For all fluid connections, fluorinated ethylene propylene (FEP) polymer tubing with an inner diameter of $500 \mu \mathrm{m}$ was used (IDEX Health \& Science). An organic to aqueous flow rate ratio, $R$, of $0.9 \pm 0.1$ was achieved by a two-step wet-etching procedure, which generates different channel cross-sections for the aqueous and organic phase streams [30]. Flow was driven by pressurised nitrogen gas (80-500 kPa) via a pressure pump (Mitos P-pump, Dolomite). Feed pressures were equal for both liquid phases. The stability of the flow in the microchip was monitored by optical microscopy (Dino-Lite Premier digital microscope) and organic phase viscosity was inferred from the measured flow rate ratio. The viscosity of the organic phase was independently measured with a manual glass viscometer (Cannon-Manning semi-micro viscometer) for comparison with microfluidics results. 


\section{Results and Discussion}

This section is organised as follows: First, we discuss the leaching of the REO ore concentrate and the composition of the resultant leach solution, including the effect of $\mathrm{pH}$ adjustment to remove base metals (specifically Fe and Al). Second, we consider the effect of fluid properties and channel geometry on the organic/aqueous flow rate ratio in the microfluidic chip. Third, the selectivity and extraction rates obtained from the HREE/LREE 'split' experiments are presented. We quantify the first-order rate constants for each element and compare micro and bulk methods. Finally, we compare the surface to volume ratios for the micro and bulk extraction systems as a possible reason for the differences in extraction rates observed.

\subsection{Leachate Composition}

Several leach solutions were derived from the REO ore concentrate by acid digestion and subsequently adjusted to $\mathrm{pH} 2,3.2,4$, or 6 . The goal of the $\mathrm{pH}$ adjustment was to remove iron and aluminium present in solution, as it can be co-extracted by Cyanex ${ }^{\circledR} 572$. In our case, about $0.1 \mathrm{~g} / \mathrm{L} \mathrm{Fe}$ were found in the original leach solution. Raising $\mathrm{pH}$ leads to its precipitation as hydroxides, which are then removed by filtration. One concern with this approach is the possibility of major losses of REEs or significant changes in the relative concentrations of heavy and light REEs in solution. The concentration of all REEs remaining in the leach solution after $\mathrm{pH}$ adjustment and filtration of the precipitate are shown in Figure 2. The concentration of every REE decreases with increasing $\mathrm{pH}$, largely due to the additional filtration required to remove solids; however the relative concentration of HREEs and LREEs remains unchanged up to $\mathrm{pH} 3.2$, as shown in Figure 3.

Complete removal of Fe is first achieved at $\mathrm{pH} 3.2$ (Figure 3) meaning that precipitation at higher $\mathrm{pH}$ is not necessary. In addition, the ratio of HREEs to LREEs did not change significantly up to $\mathrm{pH} 4$, so that the relative composition at $\mathrm{pH} 3.2$ reflects the composition of the original leach solution (prior to Fe removal). 


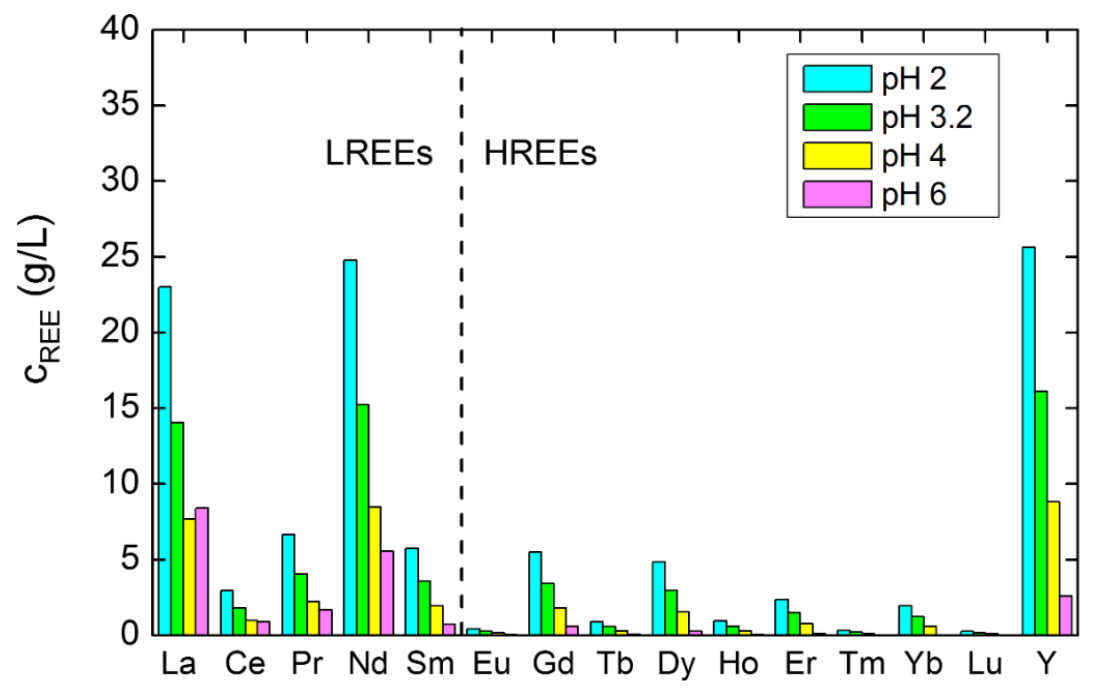

Figure 2. Concentration of REEs in the leach solution after pH adjustment to 2, 3.2, 4, and 6 after filtration of precipitate.

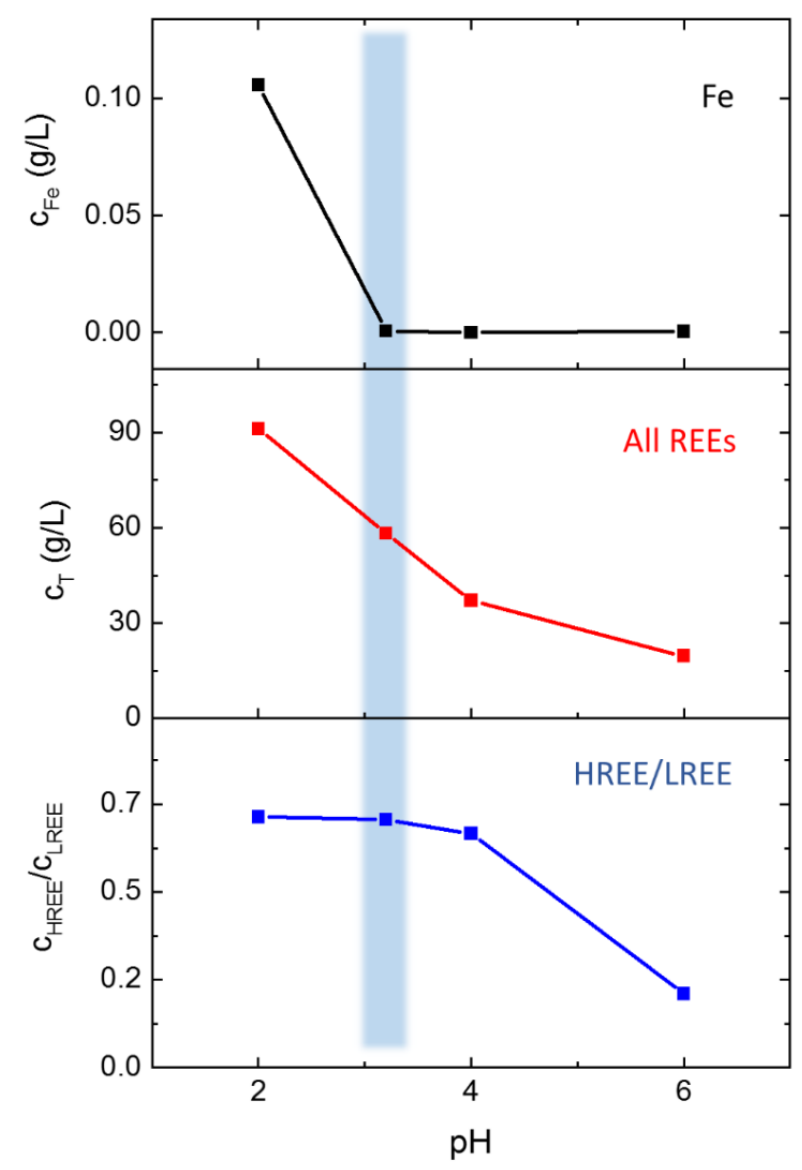


Figure 3. Concentration of iron, all REEs, and ratio of HREEs (shown together with Y) to LREEs after filtration of iron hydroxide precipitates from the leach solutions adjusted to various $\mathrm{pH}$ values $(\mathrm{pH} 2$, $3.2,4,6)$. The blue shading indicates the $\mathrm{pH}$ used for micro and bulk extractions in this study.

\subsection{Organic/Aqueous Flow Rate Ratio}

An important parameter in solvent extraction is the phase ratio, which in microSX is simply the

organic/aqueous flow rate ratio achieved, $R=\frac{Q_{\text {org }}}{Q_{a q}}$. The precise value of $R$ is determined by the microchannel geometries and the fluid viscosities, as the inlet pressures for the two phases are set to be equal in these experiments. The well-known Hagen-Poiseuille equation governs the relationship between flow resistance (defined by channel geometry and liquid viscosity), flow rate, and pressure drop. In our experiments, we fix the feed pressures for both liquid phases to be equal, which allows us to write:

$$
R=\frac{\mu_{a q}}{\mu_{o r g}} \frac{D_{o r g}^{4}}{D_{a q}^{4}}
$$

where $\mu$ is the dynamic viscosity of the liquid and $D$ is the hydraulic diameter of the channel, with subscripts referring to the organic (org) and aqueous (aq) phases. This relationship holds where channel dimensions are not vastly different [18]. In the special case where the channel cross-sections are identical, $R$ is simply $\mu_{a q} / \mu_{\text {org }}$, i.e. only dependent on the viscosities of the two liquids.

The viscosity of the organic phase containing different amounts of Cyanex ${ }^{\circledR} 572$ extractant is given in Table 1. Equation 2 predicts that the relative flow rate of organic and aqueous liquids through identical microfluidic channels at equal feed pressures can be used to verify the viscosity of one liquid when that of the other liquid is known. This verification was carried out for microchannels with etch depths, $d_{o r g}$ and $d_{a q}$, equal to $41 \mu \mathrm{m}$ (Fig. 4). The viscosities estimated from microfluidics are in good agreement with the measured ones. In this work we use an organic phase consisting of 33 v/v \% Cyanex ${ }^{\circledR} 572$ in Shellsol D70 which is about 3 times more viscous than pure water.

For the microSX experiments reported here, the microchannel etch depths were adjusted to achieve a flow rate ratio $R$ of approximately one for the known viscosity ratio. In practice, this meant 
that the channels were etched to $d_{a q}=39$ and $d_{\text {org }}=55 \mu \mathrm{m}$, see Figure 4 , which gave $R \sim 0.9$ in our experiments. The microfluidics values reported in Table 1 assume that the viscosity is unchanged during extraction. We have confirmed experimentally that this assumption is valid; the viscosity of the loaded organic phase at $90 \%$ REE loading was unchanged ( $\mu_{\text {org }}=3.0 \mathrm{mPas}$ ).

Table 1. Dynamic viscosities $\mu$ of different concentrations of Cyanex ${ }^{\circledR} 572$ in ShellSol D70.

\begin{tabular}{ccccc}
\hline Cyanex 572 & \multicolumn{3}{c}{ Cannon-Manning (Bulk) } & \multicolumn{3}{c}{ Microfluidics } \\
\cline { 2 - 5 } Concentration & $\mu_{\text {org }}$ (mPa.s) & $\frac{\mu_{a q}}{\mu_{\text {org }}}$ & $\mu_{\text {org }}$ (mPa.s) & $\frac{Q_{\text {org }}}{Q_{a q}}=\frac{\mu_{a q}}{\mu_{\text {org }}}$ \\
\hline 10 & 2.3 & 0.44 & 1.9 & 0.55 \\
20 & 2.4 & 0.41 & 2.2 & 0.48 \\
33 & 3.0 & 0.33 & 3.2 & 0.34 \\
50 & 5.9 & 0.17 & 5.3 & 0.19 \\
\hline
\end{tabular}

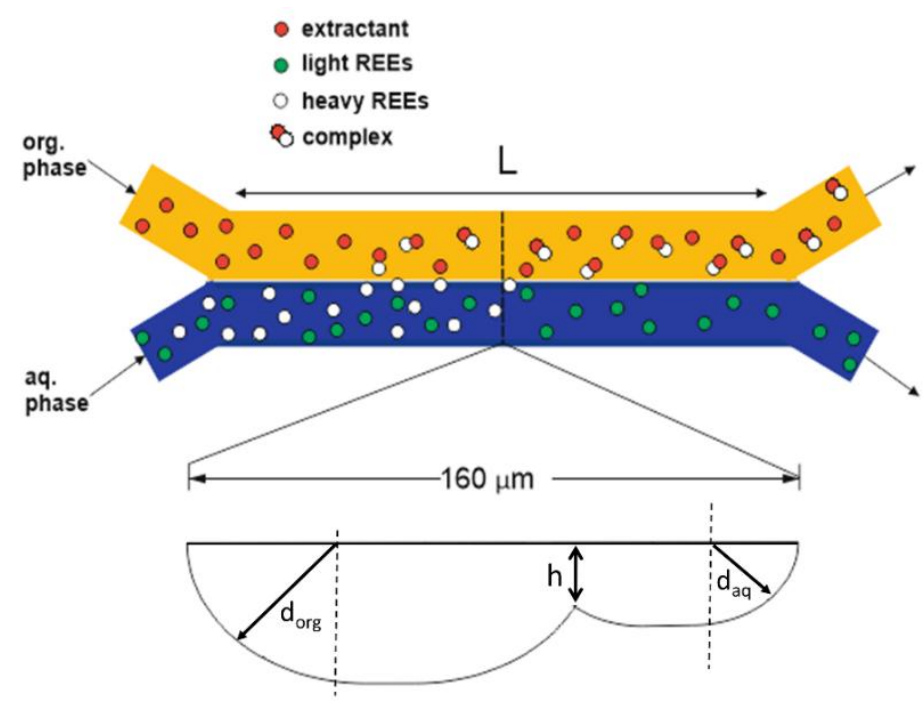

Figure 4. Schematic of the channels carrying the organic (yellow) and aqueous (blue) phases, illustrating the selective extraction of HREEs (white) by an extractant (red). The LREEs (green) remain 
in the aqueous stream. The channels have different depths, as shown in the cross-section with dimensions labelled.

\subsection{Selectivity for HREEs}

We now consider the selectivity for HREEs over LREEs in an extraction that represents a commonly used first 'split' in a REE refinery. After removal of Fe, the leach solution was adjusted to $\mathrm{pH} 0.7$ and fed into both microfluidic and bulk extractions. This initial pH 0.7 was chosen to achieve a HREE/LREE split, based on the extraction equilibria reported for Cyanex ${ }^{\circledR} 572$ by the manufacturer [7]. Figure 5 shows the micro and bulk extraction results for the REEs in the leach solution, after $10 \mathrm{~s}$ contact time. Bulk extraction results at equilibrium, i.e. 5 min, are shown for comparison.

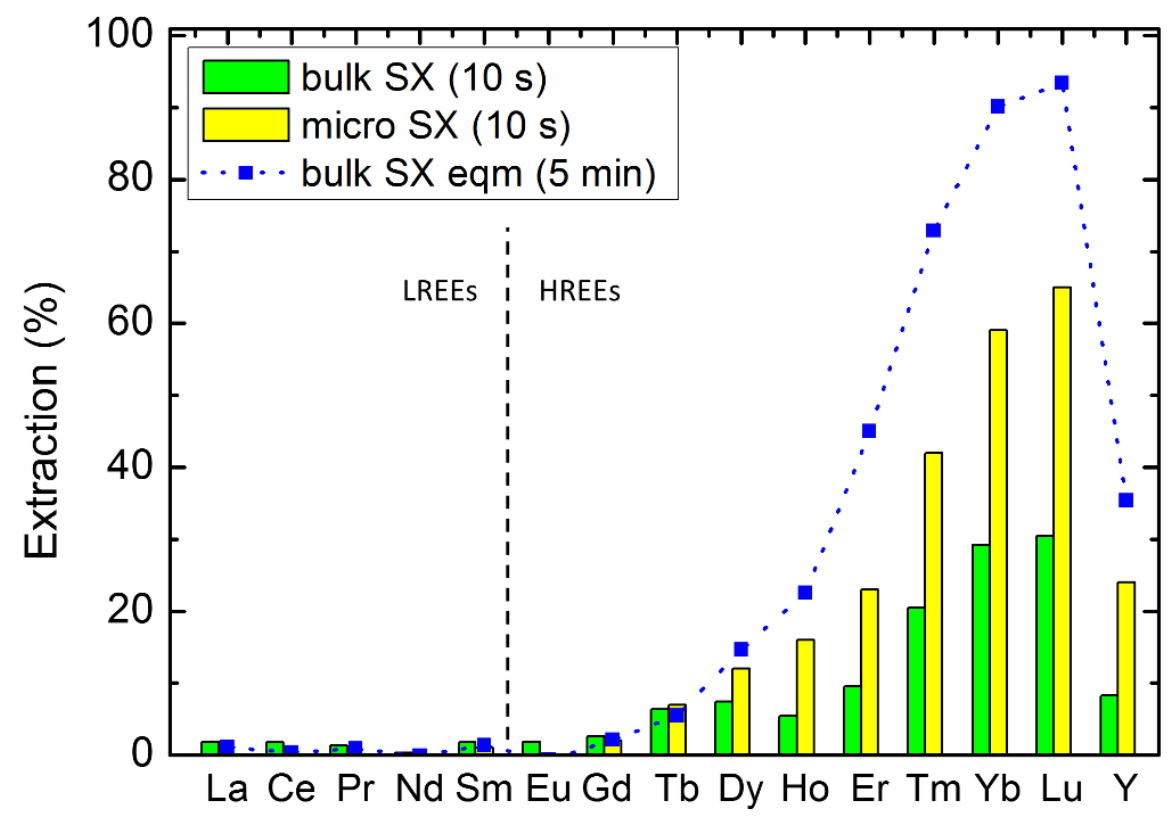

Figure 5. Comparison of micro (yellow) and bulk (green) SX of REEs from the mixed REO leach solution (initial pH 0.7) after a contact time of $10 \mathrm{~s}$ and at equilibrium (determined from bulk SX; blue points). The REEs are listed in order of increasing atomic number (except for $\mathrm{Y}$ ). 
From Figure 5 it is clear that the LREEs ( $\mathrm{La}, \mathrm{Ce}, \mathrm{Pr}, \mathrm{Nd}$ and $\mathrm{Sm}$ ) were not extracted under the chosen conditions using either bulk or microfluidic extraction. That was true at both short times (10 s) and at equilibrium (5 min). In the HREEs group, less than $5 \%$ of Eu and Gd were extracted, while the extraction of Tb through to Lu increased steadily with atomic number. Yttrium is also extracted significantly (up to $35 \%$ ), as though it is a HREE with extraction characteristics similar to Er.

Comparing the results for $10 \mathrm{~s}$ contact times, we observed that the total amount of HREEs extracted using the microfluidic extraction method was double that achieved in the bulk extraction. The total amount of HREEs extracted is given in Table 2. When compared to the amount extracted at equilibrium (bulk), the extraction is found to be $66 \%$ complete after $10 \mathrm{~s}$ contact time in the microchip and 31\% complete after $10 \mathrm{~s}$ in the bulk extraction. Possible reasons for the improved rate of extraction in the microchips are discussed later in this paper. However, it is anticipated that the difference is due to a physical, rather than chemical, phenomenon because the chemistry should not be affected greatly by the mechanism of liquid-liquid contact. It should also be noted that the loading limit in the organic phase (0.1 M REEs) is reached when the REE concentration in the aqueous phase decreases by $0.09 \mathrm{~mol} / \mathrm{L}$ due to the specific organic/aqueous phase ratio used in this study $(R=0.9)$. Thus, for bulk SX at equilibrium ( $300 \mathrm{~s}$ ) the loading limit is reached, but not exceeded. This is a critical consideration for the successful operation of the microfluidic chip, since precipitation of organic phase REE-Cyanex ${ }^{\circledR} 572$ complexes (which may foul the microchannel walls) can occur beyond the loading limit.

Table 2. Comparison of microfluidic and bulk extractions after $10 \mathrm{~s}$ of contact time, relative to bulk equilibrium (referred here to bulk after $300 \mathrm{~s}$ of contact time).

\begin{tabular}{lllll}
\hline \multirow{2}{*}{ Extraction } & Contact & Extracted & Extracted & Relative to \\
& Time & HREEs & HREEs & Equilibrium \\
Method & {$[\mathrm{s}]$} & {$[\mathrm{g} / \mathrm{L}]$} & {$[\mathrm{mol} / \mathrm{L}]$} & {$[\%]$} \\
& & & & \\
\hline
\end{tabular}




\begin{tabular}{lllll}
\hline Bulk & 10 & 3.5 & 0.028 & 31 \\
Microfluidic & 10 & 7.3 & 0.059 & 66 \\
Bulk & 300 & 10.7 & 0.090 & 100 \\
\hline Note: Maximum loading of $1 \mathrm{M}$ Cyanex $^{\circledast}$ & 572 is 0.1 mol/L REEs [5].
\end{tabular}

The chemistry of the extraction results in liberation of $\mathrm{H}^{+}$from the organic to the aqueous phase (see Eqn. (1)), thus decreasing the aqueous phase $\mathrm{pH}$ as the reaction progresses and favouring extraction of heavier REEs. Based on the stoichiometry of the extraction chemistry and the initial pH of the aqueous solution (0.7), we are able to estimate the final $\mathrm{pH}$ for the three different results shown in Table 2. We find that the final $\mathrm{pH}$ should be $0.55,0.42$, and 0.33 for the bulk (10 s), micro (10 s), and bulk (300 s) cases, respectively; however, due to the small sample volumes, this could not be directly measured. These calculated $\mathrm{pH}$ values are in good agreement with the estimated $\mathrm{pHs}$ at the extraction equilibria reported by Cytec Industries for Cyanex ${ }^{\circledR} 572$.

\subsection{Extraction Rate}

The rate of extraction is important for practical use of microSX because the flow rate is inversely proportional to the contact time for a given channel geometry and, thus, determines the volumetric throughput of the device. MicroSX enables precise control over the contact times (much less than 1 s) by adjusting flow rates and using chips containing extraction channels of different length (383 mm and $129 \mathrm{~mm}$ ). The progress of the extraction is shown in Figure 6 for each of the HREEs (LREEs were not significantly extracted, see Figure 5). The shortest reliable contact time achieved for manual sample collection from the bulk SX experiment was $5 \mathrm{~s}$. The reported contact times for the bulk SX experiments excludes any extraction that occurs during phase separation, which means that these results may slightly over-estimate extraction for the shortest time-scales. In contrast, microSX is capable of achieving contact times less than $1 \mathrm{~s}$ and phase separation is instantaneous. 
The bulk extraction results were analysed by fitting the residual REE concentration in the aqueous phase, $c_{R E E}$, with a first-order rate equation: $c_{R E E}=\alpha \exp (-k t)+\beta$, where $k\left[\mathrm{~s}^{-1}\right]$ is the rate constant, $t[\mathrm{~s}]$ is contact time, $\alpha$ is the REE concentration decrease due to extraction, and $\beta$ is the aqueous concentration of REE when extraction is complete $\left(c_{R E E}\right.$ at $\left.t \rightarrow \infty\right)$. In the microfluidic case, the contact times were not long enough to reach equilibrium. Then, by fixing $\beta$ to the value found in bulk equilibrium extraction, the microSX results were fitted using the above equation with only two parameters: $k$ and $\alpha$. The fits were in reasonable agreement in both cases; however, the rate constants were consistently much greater for microSX, see Figure 7. The greatest difference was observed for HREEs with the highest atomic number, i.e. Lu and $\mathrm{Yb}$, where the microSX rate constants were more than triple that observed in bulk SX. 

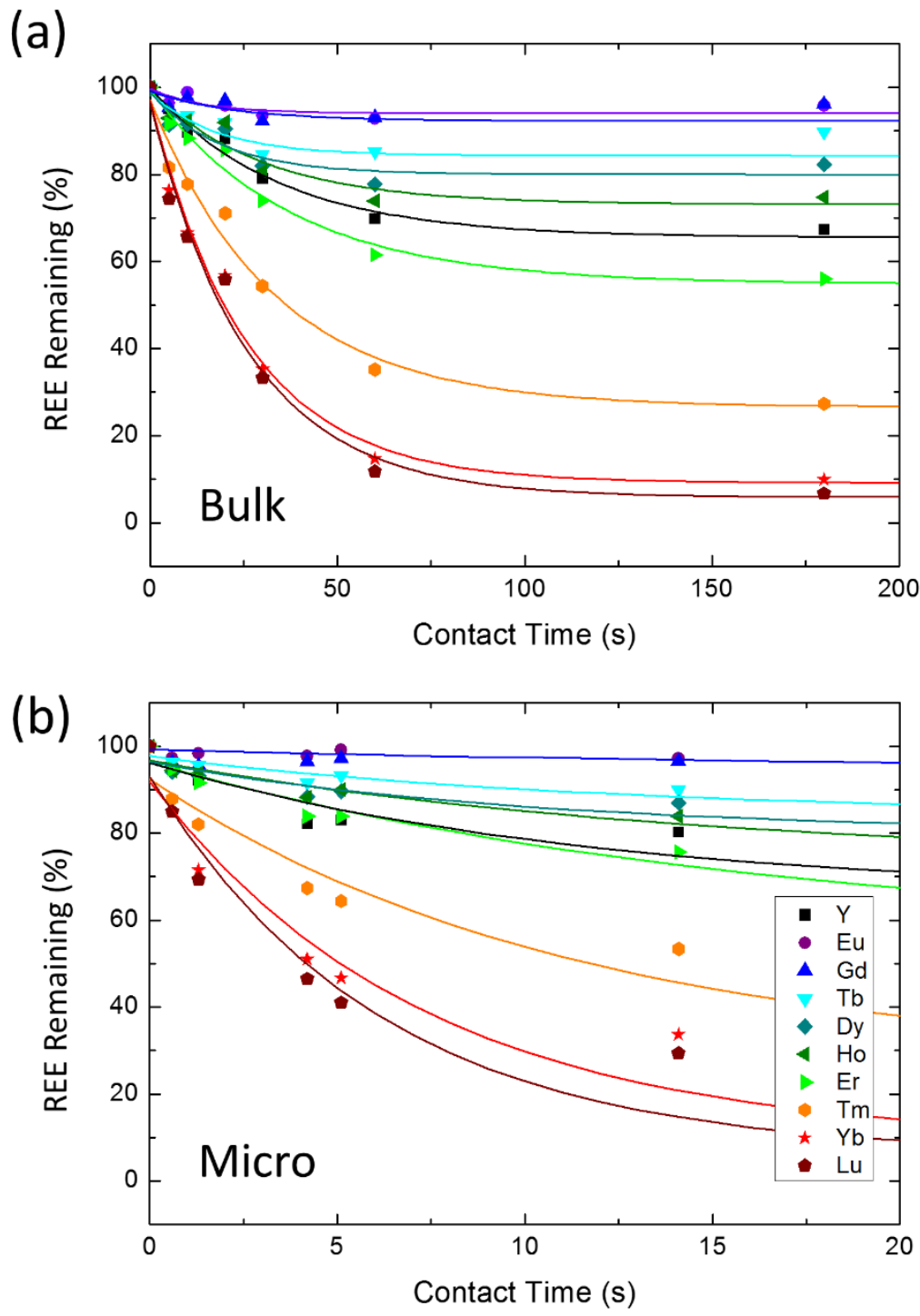

Figure 6. Extraction progress versus contact time for (a) bulk and (b) microfluidic extractions. Note that the two plots differ by an order of magnitude difference in time-scales. Lines are best fits to the experimental results for first order kinetics. In (b), the fits are forced through the equilibrium extraction obtained from bulk extraction (at $5 \mathrm{~min}$ ).

A key factor for the extraction rate is the surface available for extraction to take place. Where the surface-to-volume ratio $(\mathrm{S} / \mathrm{V})$ is increased, one can expect that the extraction rate will also be increased due to shorter diffusion lengths and more liquid-liquid interface for the extraction 
chemistry to occur. In the microchannel, the surface to volume is determined by stream geometries alone; flow rate, interfacial tension, and contact time are not important. Thus, $S / \mathrm{V}$ for microSX is easily estimated. As shown in Figure 4, the position of the liquid-liquid interface is controlled, in part, by a guide structure which is approximately $2 \mu \mathrm{m}$ high, based on scanning electron microscopy imaging (not shown). Assuming that the liquid-liquid interface is a flat plane at this location, its height will be $37 \mu \mathrm{m}$. By considering the aqueous stream, $\mathrm{S} / \mathrm{V}$ is estimated to be $13-15 \mathrm{~mm}^{-1}$ depending on the specific shape of the liquid-liquid interface.

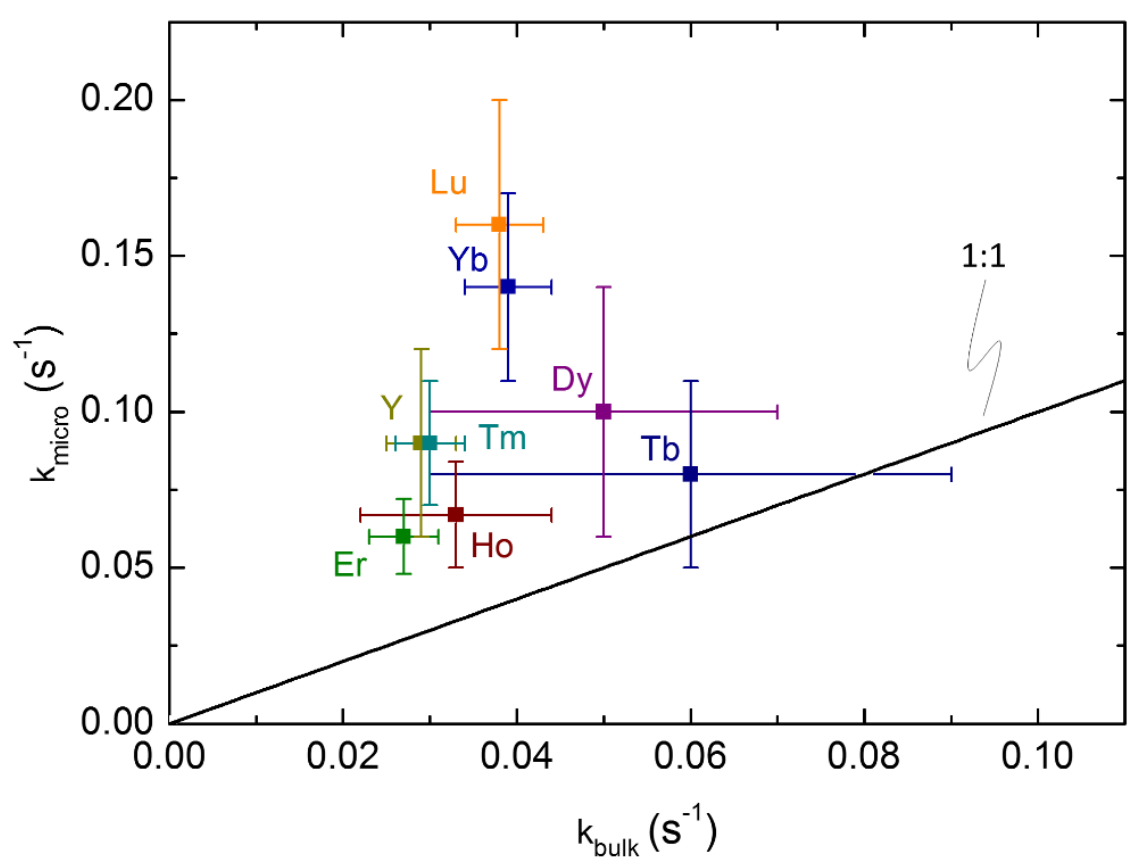

Figure 7. Comparison of rate constants determined for bulk and microfluidic extractions for REEs that are extracted significantly (i.e. HREEs excluding $\mathrm{Ga}$ and $\mathrm{Eu}$ ). The line represents equal rate constants. 


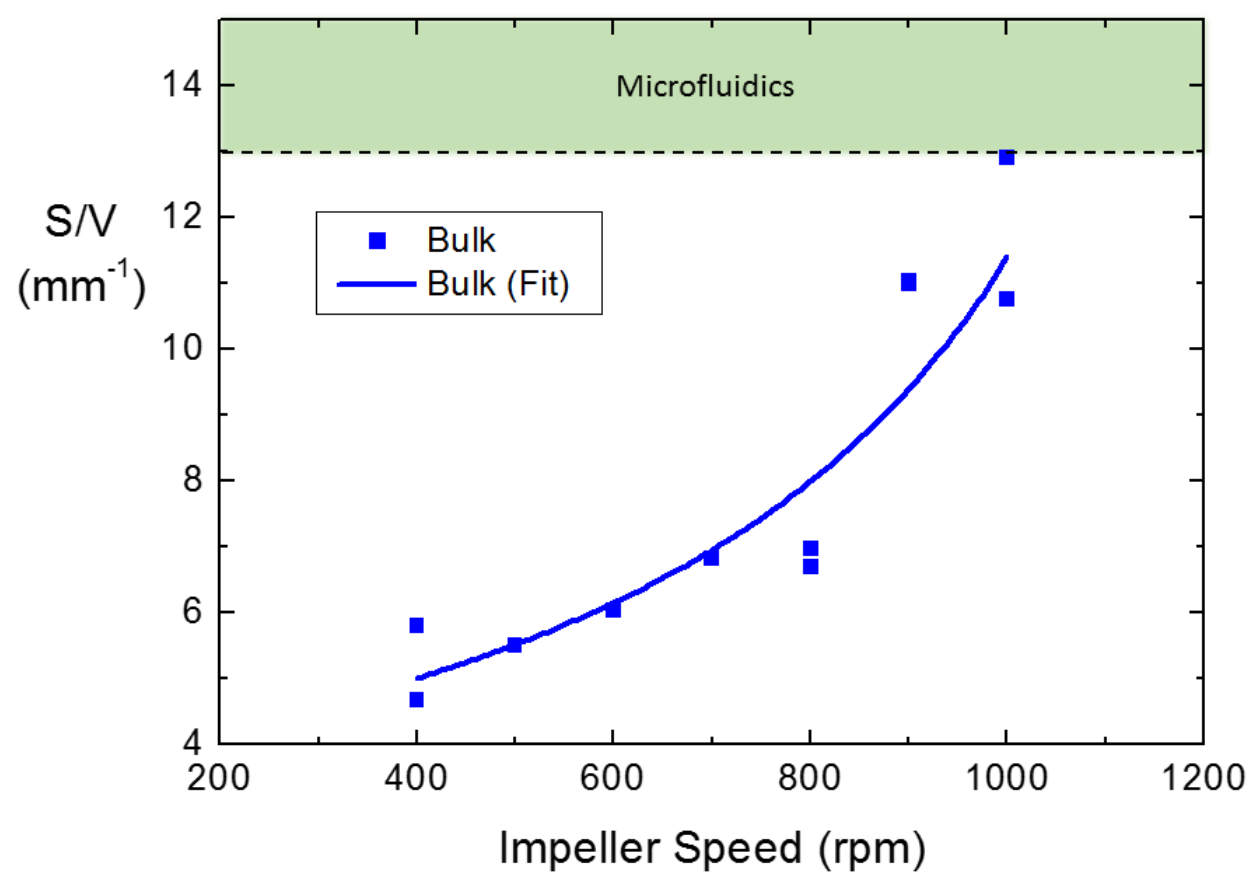

Figure 8. Surface-to-volume ratio plotted against the impeller speed. The shaded region indicates the estimated surface-to-volume ratio for the microSX chip used (i.e. disregard impeller speed).

To correctly compare the bulk and microfluidic extractions, the bulk SX S/V is also required. Therefore, we carried out bulk SX experiments and estimated the average droplet size from images taken through the beaker. Distortion was minimised by analysing images of droplets located near the centre of the beaker (where the optical distortion was smallest). The trend in our experiments (a negative power 0.9 obtained from a log-log plot of droplet size versus agitator speed) is in agreement with literature reports where the power varied between 0.75 and 1.5 as a function of the depth of immersion of the impeller [31]. The droplet size decreased from $1.3(400 \mathrm{rpm})$ to $0.5 \mathrm{~mm}(1000 \mathrm{rpm})$, which is consistent with the previous literature [32]. For the impeller speed used in this study (800 $\mathrm{rpm}$ ), the average droplet diameter was $0.8 \mathrm{~mm}$ (measured) or $0.9 \mathrm{~mm}$ (from best fit), which corresponds to approximately $\mathrm{S} / \mathrm{V}=7 \mathrm{~mm}^{-1}$. This is half that in the microSX experiments $\left(13-15 \mathrm{~mm}^{-1}\right)$ and is able to account for the difference between microSX and bulk SX rate constants for all HREEs, 
except $\mathrm{Lu}$ and $\mathrm{Yb}$. Thus, we conclude that the higher $\mathrm{S} / \mathrm{V}$ ratio is the major contributor to the faster extraction rates observed in this study.

\section{Conclusion}

Extraction rates and selectivity for HREEs were studied using bulk and microfluidic extraction methods. The microfluidic flow of co-flowing streams was designed to give an organic/aqueous flow rate ratio of 0.9 , based on the relative viscosity of the streams and the chosen microchannel geometries. A mixed REO ore concentrate was used to prepare leach solutions that mimic what is typically found in industrial REE extraction circuits. Selective extraction of HREEs from LREEs was achieved for an aqueous feed $\mathrm{pH}$ of 0.7 . For every element extracted, the microfluidic method extracted more ( $66 \%$ of equilibrium) than the bulk method ( $31 \%$ of equilibrium) after $10 \mathrm{~s}$ contact time. The rate constants obtained for microSX were approximately double that of the bulk SX, with $\mathrm{Lu}$ and $\mathrm{Yb}$ about three times greater. The faster microfluidic extraction rates can be reasonably accounted for by the higher surface-to-volume ratio (estimated at twice that observed in bulk SX), except for Lu and $\mathrm{Yb}$ which extracted according to rate constants that were three times greater than those observed for bulk SX. The ability to resolve extraction progress with sub-second resolution and control critical factors such as surface-to-volume ratios provides a powerful platform for understanding extraction chemistry and mass transport. Where parallelisation (numbering-up) proves practicable, the envisaged microSX REE refineries may be achievable due to the relatively small throughputs involved (compared to, for example, base metal circuits). In addition, microSX may yield distinct advantages if the method can minimise phase separation times in complex REE extraction circuits containing hundreds of extract, scrub, and strip stages. 


\section{Acknowledgements}

Financial support from the US Army Research Laboratory via Prime Award W911NF-10-2-0098, the Government of South Australia (PRIF IRGP18), and the University of South Australia, is gratefully acknowledged. Part of this work was performed at the SA node of the Australian National Fabrication Facility, supported by the National Collaborative Research Infrastructure Strategy. Technical assistance from Dipankar Chugh and Donghoon Chang is warmly acknowledged.

\section{References}

[1] M. Humphries, Rare Earth Elements: The Global Supply Chain Congressional Research Service, (2013).

[2] F. Xie, T.A. Zhang, D. Dreisinger, F. Doyle, A critical review on solvent extraction of rare earths from aqueous solutions, Miner Eng, 56 (2014) 10-28.

[3] G.P. Hatch, Dynamics in the Global Market for Rare Earths, Elements, 8 (2012) 341-346.

[4] K.N. Han, J.J. Kellar, W.M. Cross, S. Safarzadeh, Opportunities and challenges for treating rareearth elements, Geosyst. Eng., 17 (2014) 178-194.

[5] N. Haque, A. Hughes, S. Lim, C. Vernon, Rare Earth Elements: Overview of Mining, Mineralogy, Uses, Sustainability and Environmental Impact, Resources, 3 (2014) 614.

[6] Cytec, CYANEX ${ }^{\circledR} 572$ Solvent Extraction Reagent, Product Data Sheet, (2013).

[7] Y.L. Wang, F.J. Li, Z.Y. Zhao, Y.M. Dong, X.Q. Sun, The novel extraction process based on CYANEX (R) 572 for separating heavy rare earths from ion-adsorbed deposit, Sep. Purif. Technol., 151 (2015) 303-308.

[8] G.M. Ritcey, A.W. Ashbrook, Solvent extraction: principles and applications to process metallurgy, Elsevier Scientific Pub. Co., 1979. 
[9] C. Priest, S.F. Hashmi, J.F. Zhou, R. Sedev, J. Ralston, Microfluidic Solvent Extraction of Metal Ions from Industrial Grade Leach Solutions: Extraction Performance and Channel Aging, J Flow Chem, 3 (2013) 76-80.

[10] D. Ciceri, J.M. Perera, G.W. Stevens, The use of microfluidic devices in solvent extraction, J Chem Technol Biot, 89 (2014) 771-786.

[11] H. Nagai, N. Miwa, M. Segawa, S.-i. Wakida, K. Chayama, Quantification of $\operatorname{Ag}(I)$ and kinetic analysis using ion-pair extraction across a liquid/liquid interface in a laminar flow by fluorescence microscopy, J. Appl. Phys., 105 (2009) 102015.

[12] M. Tokeshi, T. Minagawa, T. Kitamori, Integration of a microextraction system - Solvent extraction of a Co-2-nitroso-5-dimethylaminophenol complex on a microchip, J Chromatogr A, 894 (2000) 19-23.

[13] T. Minagawa, M. Tokeshi, T. Kitamori, Integration of a wet analysis system on a glass chip: determination of $\mathrm{Co}(\mathrm{II})$ as 2-nitroso-1-naphthol chelates by solvent extraction and thermal lens microscopy, Lab Chip, 1 (2001) 72-75.

[14] C. Priest, J.F. Zhou, R. Sedev, J. Ralston, A. Aota, K. Mawatari, T. Kitamori, Microfluidic extraction of copper from particle-laden solutions, Int. J. Miner. Proc., 98 (2011) 168-173.

[15] K. Morita, T. Hagiwara, N. Hirayama, H. Imura, Extraction of $\mathrm{Cu}(\mathrm{II})$ with Dioctyldithiocarbamate and a Kinetic Study of the Extraction Using a Two-Phase Microflow System, Solvent Extr. Res. Dev. Japan, 17 (2010) 209-214.

[16] C. Priest, J.F. Zhou, S. Klink, R. Sedev, J. Ralston, Microfluidic Solvent Extraction of Metal Ions and Complexes from Leach Solutions Containing Nanoparticles, Chem. Eng. Technol., 35 (2012) 13121319.

[17] L.Q. Yang, Y.C. Zhao, Y.H. Su, G.W. Chen, An Experimental Study of Copper Extraction Characteristics in a T-Junction Microchannel, Chem. Eng. Technol., 36 (2013) 985-992.

[18] F.H. Kriel, G. Holzner, R.A. Grant, S. Woollam, J. Ralston, C. Priest, Microfluidic solvent extraction, stripping, and phase disengagement for high-value platinum chloride solutions, Chem. Eng. Sci., 138 (2015) 827-833. 
[19] C.Y. Yin, A.N. Nikoloski, M.W. Wang, Microfluidic solvent extraction of platinum and palladium from a chloride leach solution using Alamine 336, Miner Eng, 45 (2013) 18-21.

[20] K.P. Nichols, R.R. Pompano, L. Li, A.V. Gelis, R.F. Ismagilov, Toward Mechanistic Understanding of Nuclear Reprocessing Chemistries by Quantifying Lanthanide Solvent Extraction Kinetics via Microfluidics with Constant Interfacial Area and Rapid Mixing, J Am Chem Soc, 133 (2011) 1572115729.

[21] F. Kubota, J.I. Uchida, M. Goto, Extraction and separation of rare earth metals by a microreactor, Solvent Ext. Res. Dev. Japan, 10 (2003) 93-102.

[22] S. Nishihama, Y. Tajiri, K. Yoshizuka, Sepration of lanthanides using micro solvent extraction system, Ars Separatoria Acta, 4 (2006) 18-26.

[23] S.H. Yin, L.B. Zhang, J.H. Peng, S.W. Li, S.H. Ju, L.H. Zhang, Microfluidic solvent extraction of La(III) with 2-ethylhexyl phosphoric acid-2-ethylhexyl ester (P507) by a microreactor, Chem Eng Process, 91 (2015) 1-6.

[24] G. Helle, S. Roberston, S. Cavadias, C. Mariet, G. Cote, Toward numerical prototyping of labs-onchip: modeling for liquid-liquid microfluidic devices for radionuclide extraction, Microfluid Nanofluid, 19 (2015) 1245-1257.

[25] G. Helle, C. Mariet, G. Cote, Liquid-liquid extraction of uranium(VI) with Aliquat (R) 336 from $\mathrm{HCl}$ media in microfluidic devices: Combination of micro-unit operations and online ICP-MS determination, Talanta, 139 (2015) 123-131.

[26] M. Yamamoto, S. Taguchi, S. Sato, N. Surugaya, Evaluation of plutonium(IV) extraction rate between nitric acid and tri-n-butylphosphate solution using a glass chip microchannel, J Sep Sci, 38 (2015) $1807-1812$.

[27] A. Abou-Hassan, O. Sandre, V. Cabuil, Microfluidics in Inorganic Chemistry, Angew Chem Int Edit, 49 (2010) 6268-6286.

[28] F.H. Kriel, C. Priest, J. Ralston, L. Parkinson, S. Woollam, N. Plint, R.A. Grant, P. Ash, Extraction and throughput for micro-solvent extraction of platinum using a single chip and a multi-chip module, in: International Solvent Extraction Conference Würzburg, Germany, 2014. 
[29] M. Kraume, A. Gäbler, K. Schulze, Influence of Physical Properties on Drop Size Distribution of Stirred Liquid-Liquid Dispersions, Chem Eng Technol, 27 (2004) 330-334.

[30] A. Hibara, S. Iwayama, S. Matsuoka, M. Ueno, Y. Kikutani, M. Tokeshi, T. Kitamori, Surface modification method of microchannels for gas-liquid two-phase flow in microchips, Anal Chem, 77 (2005) 943-947.

[31] F.B. Sprow, Drop size distributions in strongly coalescing agitated liquid-liquid systems, AIChE J., 13 (1967) 995-998.

[32] D.E. Leng, R.V. Calabrese, Immiscible Liquid-Liquid Systems, in: Handbook of Industrial Mixing, John Wiley \& Sons 2004, pp. 639-753. 